\title{
LA INTELIGENCIA Y EL PENSAMIENTO CREATIVO: APORTES HISTÓRICOS EN LA EDUCACIÓN
}

\author{
Vivian María Pacheco Urbina
}

Recibido 1-X-2002 • Aceptado 12-XI-2002

Resumen: El propósito de este artículo es presentar información que permite establecer un marco conceptual y referencial de diversos autores que aportan posiciones teóricas sobre el desarrollo de la inteligencia y el pensamiento creativo en la educación. Se destacan personas que a través de la historia se han considerado inteligentes, por su acierto en la toma de decisiones y por el papel que han desempeñado en una serie de actividades, cuyas actuaciones marcan un cambio en los procesos sociales, políticos y científicos.

Se realiza un análisis sobre elementos fundamentales del pensamiento creativo desde la perspectiva de que la educación debe promover en el individuo la apertura a un mundo más amplio, lleno de nuevas oportunidades para el desarrollo de su potencial creativo, considerando que al tener confianza para aceptar los cambios, va a recibir la más extensa variedad de conocimiento y de experiencias enriquecedoras para el desarrollo personal.

En el artículo se describe la transformación paulatina del concepto de talento intelectual, el cual deja de estar limitado por el resultado de las pruebas de C.I., para incorporar las habilidades potenciales que el individuo tiene en una diversidad de áreas y que son fundamentales para realizar contribuciones a la sociedad.

Palabras clave: Potencialidades del Estudiante, Inteligencia, Creatividad.

\section{Introducción}

Las escuelas del futuro se planificarán, no sólo para aprender, sino también para pensar. Con mayor insistencia, a las escuelas y colegios de hoy, se les pide que produzcan hombres y mujeres que sepan pensar, que puedan hacer nuevos descubrimientos científicos, que puedan hallar soluciones adecuadas a los problemas mundiales más acuciantes, que no puedan ser sometidos a un lavado de cerebro; hombres y mujeres, en fin que sepan adaptarse al cambio y sepan mantener su cordura en esta época de movimiento constante. Este es el desafío creador que se plantea a la educación.

(Torrance,1977:14)

El objetivo del artículo es presentar una descripción histórica en relación con definiciones sobre la inteligencia y la creatividad, que en cada época, reflejan las características particulares, que prevalecen para determinar el rendimiento intelectual excepcional en el individuo, considerando las aptitudes y habilidades, y el potencial cognitivo.

La inteligencia, a través de la historia, es un factor de constantes investigaciones, que se han dirigido a medir y establecer los aspectos y características que en ella intervienen. Etimológicamente, inteligencia - "intus legere"- quiere decir: leer o conocer en su intimidad y en profundidad la realidad de las cosas. Este término hace referencia a un proceso cognoscitivo. 
En los inicios de 1900, en París, el psicólogo Alfred Binet define la inteligencia como la habilidad para tomar y mantener determinada dirección, como adaptabilidad a nuevas situaciones y habilidad para criticar los propios actos. Binet se interesa en realizar tests psicológicos simples; posteriormente, en 1905, desarrolla las pruebas de naturaleza más compleja, con la finalidad de establecer un procedimiento práctico de clasificación psicológica, las cuales, con el transcurso de los años, se utilizan como el principal instrumento de diagnóstico mental (Baker, 1950).

En 1916, Lewis Terman, de la Universidad de Stanford, realiza una revisión de los test de Binet y publica la primera edición de estos, que se conocerían como la "Revisión de Stanford y Binet", cuya finalidad era que se aplicaran a niños escolares en Estados Unidos. Este test ha sido revisado por diversos psicólogos y en la actualidad es aplicado en varios sectores educativos (Baker, 1950). En el estudio que Terman realizó, identificó a más de 1.400 niños superdotados; este estudio longitudinal se inició en 1939 y actualmente lo continúan sus asociados.

Terman establece que, como grupo, los superdotados son superiores en variados aspectos:

Son multifacéticos y espontáneos, emocionalmente estables, tienen vocabularios adelantados, luchan por alcanzar perfección, confían en sí mismos y en sus relaciones con los demás. (Sisk, 1979:8).

En relación con este proceso, la inteligencia se define como lo que las pruebas de inteligencia miden. Esta es una definición de carácter operacional.

Woodworth coincide con Binet al definir la inteligencia como retentividad; la indica como la habilidad para utilizar hechos y actividades adquiridas, adaptándolas de forma inmediata a situaciones nuevas, por curiosidad, por interés y por deseo de saber sobre las cosas y por persistencia (Baker, 1950).
Cabezas (ref. por Benito, 1990) indica que las diferentes concepciones sobre la inteligencia se pueden, operacionalmente, sintetizar en tres:

a. La de aquellos que ponen el acento en su aspecto preferentemente no ético. Plantean la inteligencia como la capacidad de sobresalir en el ámbito del aprendizaje o de adquisición de habilidades y conocimientos.

Charles Spearman (ref. por Cabezas, 1990:159) sigue esta línea en su definición de inteligencia: "Capacidad abstractiva y relacionante de los sujetos, una actividad neogenética o productora de nuevos conocimientos".

b. Las que hacen hincapié en el aspecto más bien de comportamiento. Así, la inteligencia es la capacidad del organismo para adaptarse exitosamente a la realidad o la habilidad para poder salir airoso de situaciones consideradas complicadas y embarazosas. Buckinghan (ref. por Cabezas, 1990:160) siguiendo esta línea, define la inteligencia como: "La capacidad de actuar eficientemente en condiciones dadas".

c. La de aquellos que hacen referencia a su complejidad estructural. En este sentido, entendiendo por inteligencia el conjunto de factores y funciones que determinan el promedio de éxitos de un organismo.

Berché (1990:13) plantea la aceptación inicial como indicador de superdotación, el de una superioridad intelectual en aquellos niños con coeficiente de inteligencia superior a 130 , en la escala de inteligencia para niños, de Wechsler. El autor expone los siguientes datos: 
- Uno de cada 260 niños, tiene un Coeficiente Intelectual entre 130 y 140.

- Uno de cada 3.250 niños, tiene un Coeficiente Intelectual entre 140 y 150.

- $\quad$ Uno de cada 33.360 niños, tiene un Coeficiente Intelectual entre 150 y 160.

- Uno de cada 2 millones de individuos, tiene un Coeficiente Intelectual igual o superior a 180 .

Destaca, sin embargo, que es muy difícil indicar que 130 de coeficiente intelectual es el límite por encima del cual un individuo es talentoso, debido a que las diferencias en las puntuaciones son ínfimas (130CI-129CI), y no se pueden valorar algunas veces en el comportamiento del niño. Señala el problema de la continuidad de las puntuaciones y de la precocidad; por lo que propone la flexibilidad en esta materia y el estudio y la atención individual de cada caso.

\section{Posiciones teóricas sobre la inteligencia}

En Inglaterra, Sir Francis Galton (ref. por Freeman, 1992) concebía la inteligencia como una habilidad general que forma parte de todos los tipos de actividades cognitivas, en mayor o menor grado, que difiere de una persona a otra y, por tanto, determina el nivel general de rendimiento de cada sujeto.

Se destaca en la posición teórica de los siguientes autores, cómo la inteligencia permite que el individuo se relacione con el medio socio-cultural circundante y se enfrente a las situaciones de constante cambio que se presentan en su existencia y que le demandan una gran capacidad de adaptabilidad a circunstancias, acontecimientos y vivencias.
En la Antigua Grecia, Platón seleccionaba, desde el punto de vista educativo, a los talentos del país, para poder prepararlos de acuerdo con sus condiciones superiores. Los adiestraba en Ciencia, Filosofía y Metafísica, con la finalidad de que se convirtieran, a posteriori, en conductores del Estado. Con el propósito de que Grecia pudiera perdurar, establece este plan de selección basado en una serie de pruebas (Baker, 1950).

En las etapas de la humanidad, niños con talentos especiales han superado las habilidades comunes. Entre otros, destacan músicos como Mozart, quien a los siete años de edad había publicado cuatro sonatas; escribió la primera opereta a los doce y la primera ópera italiana a los catorce. A Beethoven, desde muy niño le agradaba la música y comenzó a recibir lecciones cuando tenía seis años. Bach escribió cada semana una cantata, aun cuando se encontrara enfermo y cansado. En la pintura, Rembrandt sentía grandes deseos de pintar desde pequeño y lucha por su vocación ante sus padres. Al igual que Miguel Ángel, pintaba en horas de escuela y se mantenía en contacto con artistas de su época. El artista Diego Rodríguez de Silva y Velázquez inicia sus estudios de pintura a los diez años y a los doce es un aprendiz formal. En Sevilla es un destacado pintor a los dieciocho años, y es un famoso artista a la edad de veinticuatro años; su creatividad y producción pictórica se mantienen durante toda su vida (García, 1985). En las letras, Goethe escribió "Saludos matutinos" en alemán, latín y griego antes de cumplir los nueve años de edad. Cuando tenía diez años, Emerson escribió "La historia de Fortus, un poema caballeresco".

En Barcelona, España, en el Museo Picasso, se exhibe la colección completa de las obras de Pablo Picasso; en su infancia y adolescencia se puede observar el extraordinario dominio de las técnicas pictóricas, así como una temprana señal de una imaginación creativa que se admira mundialmente en la madurez del artista (García, 1985). 
Jean Piaget, psicólogo suizo, a los diez años de edad (1907) publica un artículo en una revista de historia natural; realiza trabajos sobre los moluscos, que son valorados por el Director del Museo de Historia Natural de Ginebra. Años más tarde se despierta su interés por la psicología y se convierte en el ayudante de Teordoro Siman, en el laboratorio Binet de París. Son relevantes sus trabajos en psicología y en desarrollo del aprendizaje y del lenguaje.

Se puede observar que el interés por los estudiantes talentosos ha sido continuo a través del tiempo. En 1866 se establecen lineamientos para la identificación de los superdotados, los cuales se incrementan por medio de la investigación y el interés en la prueba de inteligencia de Binet, en 1905; la publicación de la Escala de Inteligencia de Stanford-Binet, en 1916.

Cox, en su obra Genetics Studies of Genius (ref. por Baker, 1950), establece los rasgos mentales de los primeros años de vida de 300 hombres geniales que habían sobresalido en las actividades que habían realizado. Los psicólogos que trabajaron en procura de establecer el Coeficiente Intelectual (C.I.) de estas personas, determinaron que, en su mayoría, habían demostrado grandes capacidades desde los inicios de su niñez.

A finales del siglo XIX, se realiza una serie de actividades en Estados Unidos para brindar oportunidades a los alumnos destacados en su rendimiento académico. En Worcester, Massachusetts, en 1901 se establece la primera clase para alumnos bien dotados, en una escuela preparatoria (Baker, 1950).

A partir de 1930, se suprimen las clases o programas especiales, se fortalecen las clases regulares y se va a enfrentar un desinterés por los talentos especiales; se dan pocos proyectos de investigación en torno a este tema.

Es en 1957, con el lanzamiento del Sputnik, que surge un interés por el papel que desempeñan para la ciencia y el mundo los individuos con talento superior; French (ref. por Maker, 1995) señala que en el período de tres años, 1956-1959, se publican más artículos sobre el tema de los superdotados que en los treinta años precedentes. Así, la supremacía de los rusos en la era espacial, provoca en los norteamericanos un interés pronunciado por investigar los mejores procedimientos para la identificación y el desarrollo del talento superior académico. Se establece un nuevo currículo orientado a fortalecer los programas de la ciencia y de la matemática. La educación se propone desarrollar el talento en el área de la ciencia y de la tecnología.

En los inicios de 1970, se dirige la atención a la lucha por los derechos civiles, por una serie de movimientos que se orientan a los que presentan discapacidad y los grupos de las minorías culturales. Se valora que, a pesar de la nueva crisis que vivía el país, ésta no se podía solucionar con sólo considerar a los superdotados y a la enseñanza por grupos de habilidad.

Esta situación tiende a modificarse a mediados de la década de 1970; se da un incremento en el interés por los superdotados y sus necesidades especiales, lo cual se evidencia con mayor fuerza en 1980, en los programas educativos, en la preparación del personal docente, así como en las oportunidades para especialización en esa área en el nivel superior. Se destaca, en un inicio, el interés de la sociedad en los individuos con talento superior, con miras a que, por medio de su potencial, puedan ayudar en el desarrollo social. Mientras que en la actualidad se enfatiza en la realización plena del potencial del individuo para el logro de sí mismo, y que contribuya con la sociedad en la medida que él considere conveniente.

L.L. Thurstone (ref. por Witting, 1985:243) establece el análisis de factores para determinar siete características básicas que parecen formar la inteligencia: 
- Comprensión verbal: Definición y comprensión de palabras.

- $\quad$ Fluidez verbal: Ser capaz de pensar palabras rápidamente.

- Numérico: Ser capaz de hacer problemas aritméticos.

- $\quad$ Espacial: Ser capaz de comprender relaciones espaciales.

- Memoria mecánica: Ser capaz de memorizar y recordar.

- Perceptual: Ser capaz de captar rápidamente las similitudes, diferencias y detalles de objetos o estímulos.

- Razonamiento: Ser capaz de comprender los principios o conceptos para resolver problemas.

En años recientes, vuelve a plantearse la idea de que existen diferentes combinaciones de inteligencias humanas. Después de ocho décadas del inicio de las pruebas de inteligencia de Alfred Binet, en 1904, Howard Gardner, propone la existencia de al menos siete inteligencias básicas, en su libro Frames of Mind (Estados de ánimo, 1983). Gardner propone que la inteligencia tiene que ver más con la capacidad para resolver problemas y de crear productos en un ambiente naturalista y rico en circunstancias (Armstrong, 1995). En su estudio denota el alcance del potencial humano, trascendiendo los límites del coeficiente de inteligencia.

Gardner (1995:33), en relación con su teoría, indica:

La teoría de las inteligencias múltiples pluraliza el concepto tradicional. Una inteligencia implica la habilidad necesaria para resolver problemas o para elaborar productos, que son de importancia en un contexto cultural o en una comunidad determinada. La capacidad para resolver problemas permite abordar una situación en la cual se persigue un objetivo, así como determinar el camino adecuado que conduce a dicho objetivo. La creación de un producto cultural es crucial en funciones como la adquisición y la transmisión del conocimiento o la expresión de las propias opiniones o sentimientos. Los problemas por resolver van desde crear el final de una historia hasta anticipar un movimiento de "jaque mate" en ajedrez. Los productos van desde teorías científicas hasta composiciones musicales.

\section{El pensamiento creativo}

En las últimas décadas, el desarrollo de nuevas investigaciones de psicólogos orientadas a comprender y desarrollar las capacidades del individuo, especialmente las del intelecto, evidencian la importancia que tiene el pensamiento creativo, cuando el individuo se enfrenta a un problema que tiene que resolver.

Las investigaciones acerca de la mente y la personalidad humana y su funcionamiento han sido el objeto de estudio de psicólogos en todas las épocas; sus aportaciones han permitido despertar el interés en el pensamiento creador. $\mathrm{Al}$ respecto, Torrance (1977:126) define el pensar creativo como:

Un proceso, el proceso de intuir vacíos o elementos necesarios que faltan; de formar ideas o hipótesis acerca de ellos, de someter a prueba estas hipótesis y de comunicar los resultados; posiblemente para modificar y someter de nuevo a prueba las hipótesis ... Esta actividad creadora mental, ha sido también definida como la iniciativa que se manifiesta en la habilidad de uno a abandonar la secuencia normal del pensamiento, para pasarse a una secuencia totalmente distinta, pero productiva.

Se plantean algunas características que parecen diferenciar a las personas creativas de las que no lo son. Así, se presenta a la persona creativa como bastante flexible en los patrones de pensamiento e interesada en ideas complejas. Además, tiene una personalidad amplia, interesada en lo inusual y tiende a ser sensible a lo estético.

Se debe considerar que un acto creativo es nuevo u original, tiene una finalidad establecida y representa una solución única a un problema determinado.

Witting (1985), en su definición de lo que es un acto creativo, considera que tiene 
propósito y productividad, y da soluciones originales a los problemas.

Se destaca el trabajo de J.P. Guilford (1959-1967), al construir el modelo de inteligencia y realizar una amplia investigación, con la finalidad de identificar las 150 habilidades separadas que predecía en este modelo, destacando con sus resultados que no existe un solo puntaje de coeficiente intelectual (C.I.) que pueda indicar la inteligencia de un individuo, debido a que hay una gran variedad de "inteligencia" (Maker, 1995).

El modelo construido por Guilford (ref. por Torrance, 1965:14) plantea la estructura del intelecto en tres dimensiones:

- Operaciones mentales.

- $\quad$ Contenido o información.

- Productos.

Guilford indica que cada habilidad tiene estas tres dimensiones y que a su vez está relacionada con otras habilidades, pero es distinta.

En este modelo teórico de la estructura del intelecto, las operaciones constituyen los tipos más amplios de procesos intelectuales o actividades. Estos tipos los clasifican en cinco, los cuales son:

\section{La cognición:}

En esta se incluyen el descubrimiento, la conciencia del objeto, el reconocimiento y la comprensión o entendimiento.

\section{La memoria:}

Se refiere a la retención o almacenamiento del saber, con algún grado de disponibilidad. Se indican dos tipos de pensamiento productivo, por medio de los cuales se produce algo de lo que se ha reconocido o memorizado.

\section{Producción divergente:}

Es la generación de conocimientos, en virtud de otros conocimientos dados, en que destaca la variedad y la cantidad de lo obtenido de una misma fuente.

\section{Producción convergente:}

Es la generación de conocimientos en la que importa en mayor grado el logro de los mejores resultados únicos o convencionalmente aceptados (así, la información dada determina en forma total la respuesta).

\section{La evaluación:}

Es tomar decisiones o formular juicios concernientes a la corrección, adaptabilidad, adecuación, conveniencia de los conocimientos, en términos de criterios de identidad, consistencia y logro de la meta propuesta.

El intelecto es definido por Guilford como: "un sistema de pensamiento y factores de memoria, funciones y procesos" (Guilford, ref. por Maker, 1995:133).

Estas operaciones de Guilford (1959) permiten la valoración de la clase de operación mental que realizan los estudiantes en el proceso de enseñanza.

Shmukler (1985) indica que la creatividad es descrita de estas tres formas: en términos del producto del esfuerzo creativo, del proceso de creatividad o de la persona que es creativa.

La primera es la que tiene mayor aceptación, debido a que se obtiene de ella un producto singular, nuevo, lo que hace destacar aspectos cognitivos de la creatividad: la originalidad, la flexibilidad, la fluidez de ideas y la sensibilidad ante los problemas.

$\mathrm{Al}$ respecto, Getzels (ref. por Landau, 1992:385) indica:

El hallazgo y la formulación de un problema es un aspecto importante de la ejecución creativa, tanto en 
arte como en ciencia. En efecto, la orientación hacia los problemas, puede ser la diferencia esencial entre los científicos y los técnicos, los artistas y los copistas.

El pensamiento creativo, se pone en acción cada vez que el individuo se encuentra ante un determinado problema, que requiere de él una resolución, que emane de un conocimiento sensible y una flexibilidad mental.

Es así como el individuo, al realizar la asociación y codificación de sucesos nuevos, los relaciona con las experiencias que ha adquirido en el pasado y que han sido significativos en su vida. En gran medida, el comportamiento inteligente secuencial permite el aprendizaje por medio del discernimiento de atributos, y desarrolla así la formación posterior de conceptos (Landau, 1985).

Este proceso puede ser afectado por las categorías asignadas previamente en el medio social, educativo o cultural en el que se desenvuelve el individuo, lo cual limita su creatividad y originalidad en la formación de nuevos conceptos. El pensamiento creativo, por lo tanto, debe extrapolar la codificación aprendida, para que el individuo pueda crear un lenguaje de codificación personal, único, innovador y que permita la interrelación de nuevos aspectos cognitivos, con lo cual logra que el pensamiento proyecte la personalidad, la emoción y creatividad del individuo.

\section{El proceso de la enseñanza creativa}

En el primer siglo de pruebas formales prevaleció el interés por la evaluación de la inteligencia individual, y fue poca la atención que se les ofreció a otras capacidades cognitivas.

En la era del post-Sputnik, las autoridades educativas concentran la atención en la importancia de la imaginación, inventiva y creatividad del individuo. Surge así la necesidad de crear instrumentos que evaluaran la creatividad o el potencial creativo.

Al respecto, Gardner (1993:170) indica:

...en su búsqueda de formas para medir la creatividad, repitieron la mayoría de los errores que se habían cometido a través de la historia de las pruebas de inteligencia. O sea, trataron de crear mediciones de las habilidades de pregunta corta y con un tiempo limitado; habilidades que creían esenciales para la creatividad, la capacidad de dar una variedad de respuestas a una pregunta (pensamiento divergente) o crear muchas asociaciones inusuales a un estímulo (fluidez de ideas).

De acuerdo con el criterio de investigadores, las pruebas para la creatividad no han llenado su principal función. Son instrumentos confiables que miden algo más que la inteligencia psicométrica, pero no pueden ser pruebas predictoras de la creatividad de un individuo, pues sus producciones se basan en un campo determinado (Gardner, 1993).

En años recientes, los investigadores se dirigen a examinar con detalle lo que sucede cuando los individuos se enfrentan con actividades de solución o de encontrar problemas. Estos estudios aportan dos resultados significativos:

a. Los individuos creativos no parecen tener a su disposición operaciones mentales que les pertenezcan sólo a ellos; los individuos creativos hacen uso de los mismos procesos cognitivos que las demás personas, pero los usan de forma eficiente y flexible, y al servicio de metas ambiciosas y, generalmente riesgosas. (Perkins, ref. por Gardner, 1993:171).

b. Los individuos altamente creativos, parece que llevan la vida de forma diferente. Están muy metidos en su trabajo y lo hacen con pasión, tienen la necesidad de hacer cosas nuevas y tienen claros sus propósitos y metas. 
Además, son muy reflexivos en sus actividades, el uso del tiempo y la calidad de lo que hacen. (Gruber, ref. por Gardner, 1993:171).

Es importante denotar los principios de la enseñanza creativa, definidos por Logan y Logan (1980:48), que resumen las características que deben considerarse en una educación promovedora del desarrollo creativo en el alumno:

a. Es de naturaleza flexible: Está en función de la singularidad de los alumnos, y estructurada de acuerdo con las diferentes capacidades, intereses e historiales sociales de los niños.

b. Requiere métodos de enseñanza indirecta: Requiere del arte de deducir, preguntar, sugerir, proporcionar pistas, indicar alternativas e integrar. Estimula las capacidades asociativas, pone en movimiento las operaciones connotativas y fomenta el pensamiento creativo.

c. Es imaginativa.

d. Fomenta el uso único de materiales e ideas: Debe ofrecer oportunidad de combinar actos y pensamiento, la experiencia y la creación, el aprendizaje y la experimentación; ello es esencial si se quiere que los niños desarrollen al máximo su potencial.

e. Favorece la relación: Que implica una interacción en la que intervienen el profesor, el alumno, el tema y una experiencia o actividad de aprendizaje particular.

f. Es de naturaleza integradora.

g. Refuerza la autodirección: El maestro sólo guía el aprendizaje, pero es el niño quien aprende. h. Implica autovaloración.

i. Comporta riesgos, pero aporta recompensas: El aprendizaje implica autorrealización.

En este contexto el papel del docente es fundamental, ya que es él quien reconoce, estimula y recompensa el talento creativo del alumno. Debe prevalecer en todo momento el espíritu creativo y original del docente, que promueva e incentive el crear, inventar, imaginar e interrogar.

En el individuo talentoso, su capacidad intelectual superior y sus capacidades especiales le permiten obtener logros en diversos campos; la valoración de estos debe integrar factores personales, familiares, educativos, socioeconómicos, ambientales, culturales, entre otros que intervienen en el desarrollo de sus potencialidades y en la obtención de un rendimiento con excelencia.

\section{Conclusiones}

$\mathrm{Al}$ promover una educación que permita el desarrollo del talento creativo de los niños, los docentes deben tener motivación para estimular una mente creativa en los alumnos.

Shmukler (1985) indica que el juego imaginativo, siendo afectivo y cognitivo a la vez, tiene una función esencial para el desarrollo y ayuda al niño a adquirir un equilibrio entre la experiencia interior y exterior, y a desarrollar una reserva de recursos, actividades y autoestima, estimulando al mismo tiempo la curiosidad y la capacidad para investigar.

Es fundamental considerar que en cualquier etapa del desarrollo del niño el juego es de sumo interés, debido al gran impulso creativo que fomenta en él; además de que este aprende a conocerse a sí mismo, mediante las experiencias que vive durante los juegos. En ellos establece relaciones con los demás niños y descubre sus 
habilidades, destrezas y necesidades. En este sentido, el ambiente y las condiciones sociales que rodean al niño son primordiales para estimular el proceso de creatividad.

Se puede enseñar a los niños de manera que utilicen sus habilidades creadoras de la mente, ya que éstas son bastante distintas de aquellas que se miden con las pruebas tradicionales de inteligencia. Así, el niño creativo requiere, para su pleno desarrollo, la oportunidad de participar activamente en el proceso de enseñanzaaprendizaje; en él expone sus ideas y fantasías, expone su iniciativa, manifiesta su curiosidad y plantea interrogantes, necesidades, inquietudes y aspiraciones.

En el desarrollo de las potencialidades del niño, se debe estimular su inventiva, su participación en la solución de problemas; proporcionando un marco de libertad y de respeto, en el que pueda manifestar sus ideas innovadoras y sus dotes de investigador.

En un proceso educativo integrador es necesario estimar los resultados obtenidos de la aplicación de: los tests de inteligencia colectiva e individuales, de rendimiento académico estandarizado, pruebas de diagnóstico; asimismo, se requiere considerar las características de la personalidad del estudiante, el talento cognitivo, las aptitudes, las habilidades, la capacidad creativa, la motivación, el desarrollo en la actividad escolar, la relación con el núcleo familiar y con el ambiente sociocultural en el que se desenvuelve la persona. Así, el fin de la educación no es sólo una preparación para la vida, sino que también es el cómo facilitar el desarrollo de actitudes creativas y abiertas al conocimiento del estudiante.

El ser humano, desde su nacimiento, se encuentra en una interacción constante con la familia y el contexto social; por lo que, para desarrollar las capacidades intelectuales, aptitudes y habilidades requiere del afecto, comprensión, atención y estímulo de las personas que comparten sus aspiraciones, expectativas y metas.
Asimismo, necesita de instituciones educativas que se interesen por estimular sus potencialidades en los marcos sociales y culturales en que se desenvuelve. Por lo tanto, se recomienda la integración de la familia, la sociedad y las instituciones educativas en proyectos que fomenten el desarrollo del talento en los estudiantes.

\section{Referencias bibliográficas}

Armstrong, Thomas. Inteligencias múltiples en el salón de clases. Association for Supervision and Curriculum Development. Alexandria, Virginia, Estados Unidos. 1995.

Baker, Harry J. Introducción al estudio de los niños sub y superdotados. Trad. de Ghioldi, Alfredo M. Segunda Parte. Editorial Kapelusz. Buenos Aires, Argentina. 1950.

Benito M., Yolanda. La identificación o diagnóstico del niño superdotado. En: Problemática del niño superdotado. Salamanca: Ediciones Amarú. 1990.

Berché C., Javier. Concepto de superdotación. En: Benito M., Yolanda. Problemática del niño superdotado. Salamanca: Ediciones Amarú. 1990.

Cabezas, Juan A. Creatividad e inteligencia. En: Benito M., Yolanda. Problemática del niño superdotado. Salamanca: Ediciones Amarú. 1990.

Freeman, Joan. Los niños superdotados. Aspectos psicológicos y pedagógicos. Trad. Illueca García, Ma. del Carmen. Editorial Santillana, S.A. Madrid, España. 1992.

García Etchegoyhen de Lorenzo, Eloísa. El niño retardado mental y el niño 
superdotado talentoso. ¿Son ellos las dos caras de una misma moneda? Montevideo: Instituto Interamericano del Niño. 1985.

Gardner, Howard. Creatividad. Five forms of creative activity: a Development perspective. Proyect Zero, Harvard Graduate Scholl of Education. Paper prepared for the Wallace National Symposium of Talent Development, University of Iowa. Estados Unidos. 1993.

Gardner, Howard. Creatividad. Progressiving in a new key. Proyect Zero, Harvard Graduate Scholl of Education. Paper prepared for the Wallace National Symposium of Talent Development, University of Iowa. Estados Unidos. 1993.

Gardner, Howard. Inteligencias múltiples. La teoría en la práctica. Ediciones Paidós. Barcelona, España. 1995.

Landau, Erika. Formulando preguntas creativas para el futuro". En Freeman, Joan. Los niños superdotados. Aspectos psicológicos y pedagógicos. Trad. Illueca García, Ma. del Carmen. Editorial Santillana, S.A. Madrid, España. 1992.

Logan y Logan. Estrategias para una enseñanza creativa. Editorial OI Kostau S.A. 1980.
Maker C., June. Educación del superdotado. Tendencias significativas. Documento proyecto: Apoyo a la atención y desarrollo de las aptitudes y talentos de los niños y niñas de escuelas públicas. Ministerio de Educación Pública. 1995.

Shmukler, Diana. Fundamentos de la creatividad: El ambiente favorecedor. En Freeman, Joan. Los niños superdotados. Aspectos psicológicos y pedagógicos. Trad. Illueca García, Ma. del Carmen. Editorial Santillana, S.A. Madrid, España. 1992.

Sisk, Dorothy A. Exposición del problema. En: Los niños superdotados. Tampa, Florida. Secretaría del Año Internacional del Niño. 1979, pág. 5-12.

Torrance, E. Paul. Cómo es el niño sobredotado y cómo enseñarle. Trad. Thomas, Juan J. Editorial Paidós. Buenos Aires, Argentina. 1965.

Torrance, E. Paul. Educación y capacidad creativa. Trad. Piqué, Jorge. Ediciones Morova, S.L. Madrid, España. 1977.

Witting, Arno F. Introducción a la psicología. Teoría y 618 problemas resueltos. Serie Schaum. Traducción Dulcey Ruiz, Elisa. Libros Mc Graw-Hill de México, S.A. De C.V. México. 1985.

Vivian María Pacheco Urbina Profesora de la Escuela de Administración Educativa de la Universida de Costa Rica 